\title{
Circulating microRNAs - a new horizon in molecular diagnosis of breast cancer
}

\author{
Hoda A. Hagrass ${ }^{1}$, Samar Sharaf ${ }^{1}$, Heba F. Pasha ${ }^{2}$, Enas A. Tantawy ${ }^{3}$, Randa H. \\ Mohamed², Rasha Kassem4 \\ ${ }^{1}$ Clinical Pathology Department, Faculty of Medicine, Zagazig University, Egypt \\ ${ }^{2}$ Medical Biochemistry Department, Faculty of Medicine, Zagazig University, Egypt \\ ${ }^{3}$ Microbiology and Immunology Department, Faculty of Medicine, Zagazig University, Egypt \\ ${ }^{4}$ Surgery Department, Faculty of Medicine, Zagazig University, Egypt
}

Correspondence to: Hoda A. Hagrass, email: huda_hagrass@yahoo.com

Keywords: Biomarker, breast cancer, microRNAs, real time PCR, diagnosis

Received: April 14, $2015 \quad$ Accepted: May 28, $2015 \quad$ Published: June 3, 2015

This is an open-access article distributed under the terms of the Creative Commons Attribution License, which permits unrestricted use, distribution, and reproduction in any medium, provided the original author and source are credited.

\section{ABSTRACT}

Background: The potential use of microRNAs (miRNAs) as ideal tumor markers has been the focus of recent research.

Objective: Our hypothesis was that circulating miRNAs are differentially expressed in pretherapeutic sera of breast cancer patients compared to controls.

Materials and Methods: Using real-time quantitative polymerase chain reaction (qPCR) analysis, levels of 5 candidate miRNAs (miR10b, miR34a, miR155, miR195 and $\mathrm{miR} 16$ ) were quantified in sera of breast cancer patients and control individuals.

Results: Levels of preoperative sera showed significant upregulation of $\mathbf{3 . 3 6}$ fold rise in miR10b $(p<0.001)$, a 2.07 fold rise in miR155 $(p=0.005)$ and remarkable over expression of 11.9 fold rise in miR195 $(p<0.001)$ of cases than controls. There was significant down regulation of miR34a $(0.032, p<0.001)$. The comparison with the clinicopathological data of the breast cancer patients revealed significant high serum level of miR155 $(p=0.004)$ and miR195 $(p=0.002)$ in patients with lymph node metastasis and higher levels of $\operatorname{miR10b}(p=0.001)$ and $\operatorname{miR155}(p<0.001)$ with distant metastasis (M1) than without metastasis (M0), in addition to significant decrease in miR34a $(p<0.001)$ level in M1 than M0 cases.

Conclusions: These findings suggest that systemic circulating miRNAs have potential use as novel biomarkers for breast cancer.

\section{INTRODUCTION}

Breast cancer (BC) is the most common cancer and the second death causing cancer in women. Every year, more than one million women are diagnosed with breast cancer and approximately 400,000 die of it [1]. The disease is also considered the most common cancer in Egyptian women and accounts for $29 \%$ of National Cancer Institute cases. The age at diagnosis is lower than in countries of Europe and North America and most of the diagnosed females are premenopausal [2].

Early diagnosis of cancer remains a challenge for clinicians; it is an important goal to reduce treatmentassociated morbidity \& mortality and to reach maximal long-term survival [3].

MicroRNAs (miRNAs) are a class of small noncoding endogenous RNA molecules, 18-25 nucleotides long. Since their discovery, the investigators have found that they play important regulatory roles in many biological and pathological processes [4].

Tumors may be caused by down-regulation of a tumor suppressor miRNA and / or overexpression of an oncogenic miRNA. Micro RNA studies in breast cancer showed their importance and potential use in tumor classification and prognosis [5].

Iorio and colleagues found that miRNAs are deregulated in cancer tissues when compared with normal breast tissues and they emphasized the role of this 
deregulation in development of breast cancer [5].

As miRNAs regulate essential aspects of tumor biology and are stable in both blood and formalin-fixed, paraffin-embedded tissue samples, their use as potential prognostic and predictive markers has evolved [6,7].

Van Schooneveld and co-investigators profiled 768 miRNAs in 84 breast cancer tissue samples and 8 normal tissue samples obtained after breast-reductive surgery [8]. Whereas, Wang and colleagues showed that the high levels of miR9 and miR200c in breast cancers might serve as molecular diagnostic markers for the disease [9].

In this study, we aimed to investigate the utility of a panel of circulating miRNAs (miR10b, miR34a, miR155 and miR195) as potential biomarkers in breast cancer, particularly in early stages of the disease, this will be the first work studying these miRNAs in Egyptian female patients with breast cancer.

\section{RESULTS}

One hundred and twenty breast cancer patients (48 premenopausal and 72 post menopausal) and 50 healthy controls were included in the analysis. Cases and controls were matched for age (mean age; $53 \pm 6.75 \& 52 \pm 8.1$ years, for patients and controls respectively, $p=0.16$ ).

\section{Demographic and clinicopathological characteristics of breast cancer patients}

All cases had histologically confirmed diagnosis (25 carcinoma in situ and 95 invasive carcinoma). Tumor grading revealed 53 cases of grades I \&II and 67 cases of grade III. Based on TNM, 50 patients were in the early stages (0\&I), while the other 70 patients were advanced (II, III and IV). Lymph node (LN) involvement was reported in 76 cases. Hormone receptors were evaluated in tissue samples: 80 cases were positive ( $+v e$ ) for estrogen receptors (ER), 40 were ER negative (-ve), 83 were progesterone receptor $(\mathrm{PR})+\mathrm{ve}, 37$ were $\mathrm{PR}-\mathrm{ve}, 76$ were HER2 -ve and 44 were HER2 +ve (Table 1).

\section{Fold changes of miR16, miR10b, miR34a, miR155 and miR195 in breast cancer patients compared to the controls}

As its expression is stable and reproducible, miR16 was chosen as an endogenous control to standardize miRNA expression. We quantified the relative expression of the four miRs (miR10b, miR34a, miR155 and miR195) in the blood of patients and controls. As shown in table 2 , the analysis revealed a significant up-regulation

Table 1: Demographic and clinicopathological characteristics of breast cancer patients.

\begin{tabular}{cc}
\hline Variable & Frequency (\%) \\
\hline Age (53 \pm 6.75$)$ years & $48(40 \%)$ \\
Premenopausal & $72(60 \%)$ \\
Post menopausal & \\
Types & $25(20.8 \%)$ \\
Carcinoma in situ & $95(79.2 \%)$ \\
Invasive carcinoma & \\
Grade & $53(44.2 \%)$ \\
I, II & $67(55.8 \%)$ \\
III & \\
Stage & $50(41.7 \%)$ \\
Early stage (0\& I) & $70(58.3 \%)$ \\
Advanced stage (II, III\& IV) & \\
ER & $40(33.3 \%)$ \\
-ve & $80(66.7 \%)$ \\
PR & \\
-ve & $37(30.8 \%)$ \\
+ve & $83(69.2 \%)$ \\
HER2 & \\
+ve & $44(36.7 \%)$ \\
-ve & $76(63.3 \%)$ \\
Lymph node & \\
-ve & $76(63.3)$ \\
+ve & $44(36.7)$ \\
\hline
\end{tabular}


Table 2: Fold changes of miR16, miR10b, miR34a, miR155 and miR195 in Breast Cancer patients compared to the control group.

\begin{tabular}{|c|c|c|c|c|c|}
\hline & miR16 & miR10b & miR34a & miR155 & miR195 \\
\hline Fold change & 1.11 & 3.36 & 0.032 & 2.07 & 11.9 \\
\hline 95\% CI & $(0.68,1.54)$ & $(2.53,4.19)$ & $(0.02,0.04)$ & $(1.14,3.01)$ & $(6.17,17.65)$ \\
\hline p-value & 0.63 & $<0.001^{*}$ & $<0.001^{*}$ & $0.005^{*}$ & $<0.001^{*}$ \\
\hline
\end{tabular}

*Significant.

in the form of 3.36 fold rise in miR10b $(p<0.001)$, a 2.07 fold rise in $\operatorname{miR} 155(p=0.005)$ and a remarkable overexpression of 11.9 fold rise in $\operatorname{miR} 195(p<0.001)$ of cases than controls. In contrast, there was a significant down regulation of miR34a $(0.032, p<0.001)$.

\section{Relationship between miRs (miR10b, miR34a, miR155 and miR195) in serum of breast cancer patients and their clinicopathological features}

The relative concentrations of serum miRs (miR10b, miR34a, miR155 and miR195) of the 120 breast cancer patients were studied in relation to their clinicopathological data. The transcript levels of miR10b $(p=0.001)$ and miR155 $(p<0.001)$ were significantly higher in sera of patients with grade (III) than in those with lower grade tumors (I \& II). Moreover, the serum level of miR 155 was significantly increased in advanced stage cases (II, III\& IV) than those in early stages $(0$ \& I) $(p=0.004)$. On the other hand, levels of miR34a were significantly down regulated in advanced stage (II, III\& IV) cases than in early stages $(0 \&$ I) $(p<0.001)$ (Table 3$)$.

In respect to the relation between miRs serum levels and lymph node involvement, we found significantly higher serum levels of miR155 $(p=0.004)$ and miR195 ( $p$ $=0.002$ ) in cases with lymph node metastasis compared to thoses with no lymph node metastasis (Table 3 ).

Remarkably, we revealed significantly higher serum levels of miR10b $(p=0.001)$ and miR155 $(p<0.001)$ in cases with distant metastasis (M1) than those with no distant metastasis (M0), in addition to a significant decrease in miR34a $(p<0.001)$ serum levels in M1 than M0 cases (Table 3 ).

Regarding ER status, circulating miR10b levels were higher in ER -ve when compared with ER +ve patients $(p=0.001)$, and no statistically significant difference was detected for other miRs.

The statistical analysis of the concentrations of the studied miRs in relation to the other clinicopathological features as well as the immunohistochemical presence of ER, PR \& HER2 did not reach any statistical significance (Table 3).

\section{DISCUSSION}

In this study, we analyzed the levels of four miRNAs (miR10b, miR34, miR155, and miR195) in serum from $120 \mathrm{BC}$ patients and 50 healthy controls. Our assay showed significantly higher serum levels of miR-10b, miR155, and miR195 in BC patients and a significantly lower serum level of miR34 in BC patients.

The role of miR10b in breast cancer has been addressed with conflicting results. Earlier studies found that miR10b was down-regulated in breast tumors compared to normal breast tissues $[5,10]$. Ma et al. and Mar-Aguilar et al. opposed these findings, and reported that miR10b was significantly higher in the serum of BC patients than controls which is in line with our results $[11,12]$. On the contrary, Heneghan and colleagues reported that the level of expression of miR10b in $\mathrm{BC}$ patients is similar to that of healthy controls [3].

With regard to miR34a, a discrepancy of its expression in diverse tumors has been described. Dutta and co-authors found a high incidence of miR34a overexpression in various tumor types, and undetectable expression in only poorly differentiated gastric adenocarcinomas and renal cell carcinomas [13]. In addition, Roth and colleagues showed high miR34a levels in the blood of breast cancer patients [14]. In contrast, it was reported that miR34a was downregulated in various cancer types including non-small cell lung carcinomas, pancreas tumor cell lines, colon carcinomas, primary neuroblastomas and breast cancer [15-17].

In the same line with our results, Mar-Aguilar et al. found that the levels of miR155 were significantly higher in serum of BC patients than in controls [12]. Upregulation of miR 155 has been observed in human primary breast cancers, and was over-expressed significantly in tumor specimens of $\mathrm{BC}[5,18,19]$. Moreover, Wang and his colleagues reported that mi-R155b was consistently up regulated in cancer samples of both tissues and matching sera compared with their controls [20]. In contrary to our results, prior studies by Heneghan et al. and Zhu et al. reported no significant difference in the level of expression of miR 155 between $\mathrm{BC}$ patients and healthy controls [3, 21].

Concerning miR195, our results confirmed the results obtained by other studies. Zhang and his colleagues identified miR195 to be significantly elevated in breast 
Table 3: Relationship between MicroRNAs' (miR10b, miR34a, miR155 and miR195) fold changes and Clinicopathological Characteristics of patients.

\begin{tabular}{|c|c|c|c|c|}
\hline Parameters & miR10b & miR34a & miR155 & miR195 \\
\hline Premenopause & $3.1 \pm 0.9$ & $0.038 \pm 0.026$ & $1.85 \pm 0.17$ & $11.8 \pm 7.8$ \\
\hline Postmenopause & $3.4 \pm 1.3$ & $0.035 \pm 0.024$ & $1.92 \pm 0.16$ & $12.6 \pm 8.8$ \\
\hline $\mathrm{p}$-value & 0.3 & 0.5 & 0.05 & 0.6 \\
\hline $\begin{array}{c}\text { Type: } \\
\text { Carcinoma in situ }\end{array}$ & $2.98 \pm 0.9$ & $0.035 \pm 0.026$ & $1.85 \pm 0.16$ & $10.8 \pm 3.6$ \\
\hline Invasive carcinoma & $3.4 \pm 1.2$ & $0.037 \pm 0.025$ & $1.9 \pm 0.18$ & $12.7 \pm 9.2$ \\
\hline $\mathrm{p}$-value & 0.1 & 0.8 & 0.2 & 0.3 \\
\hline Low grade $(\mathrm{I}, \mathrm{II})$ & $2.9 \pm 0.8$ & $0.039 \pm 0.031$ & $1.83 \pm 0.19$ & $13.2 \pm 9.98$ \\
\hline High grade (III) & $3.6 \pm 1.3$ & $0.035 \pm 0.018$ & $1.95 \pm 0.14$ & $11.6 \pm 6.85$ \\
\hline $\mathrm{p}$-value & $0.001^{*}$ & 0.4 & $<0.001^{*}$ & 0.3 \\
\hline Early stage $(0 \&$ I $)$ & $3.5 \pm 1.3$ & $0.047 \pm 0.03$ & $1.84 \pm 0.13$ & $13.9 \pm 10.06$ \\
\hline Advanced stage (II,III\&IV) & $3.1 \pm 1.04$ & $0.028 \pm 0.017$ & $1.93 \pm 0.19$ & $11.1 \pm 6.77$ \\
\hline $\mathrm{p}$-value & 0.07 & $<0.001^{*}$ & $0.004 *$ & 0.07 \\
\hline ER & & & & \\
\hline -ve & $3.8 \pm 1.2$ & $0.032 \pm 0.021$ & $1.85 \pm 0.22$ & $13.5 \pm 10.2$ \\
\hline$+\mathrm{ve}$ & $3.1 \pm 1.1$ & $0.038 \pm 0.027$ & $1.91 \pm 0.15$ & $11.7 \pm 7.3$ \\
\hline $\mathrm{p}$-value & $0.001^{*}$ & 0.3 & 0.09 & 0.2 \\
\hline $\begin{array}{l}\text { PR } \\
\text {-ve } \\
+ \text { ve }\end{array}$ & $\begin{array}{l}3.2 \pm 1.1 \\
3.3 \pm 1.1\end{array}$ & $\begin{array}{l}0.031 \pm 0.021 \\
0.038 \pm 0.026\end{array}$ & $\begin{array}{c}1.88 \pm 02 \\
1.89 \pm 0.16\end{array}$ & $\begin{array}{c}12.6 \pm 10.9 \\
12.1 \pm 7.1\end{array}$ \\
\hline p-value & 0.6 & 0.2 & 0.8 & 0.8 \\
\hline $\begin{array}{l}\text { HER2 } \\
\text {-ve }\end{array}$ & $3.1 \pm 1.1$ & $0.036 \pm 0.032$ & $1.90 \pm 0.18$ & $13.7 \pm 11.4$ \\
\hline$+\mathrm{ve}$ & $3.4 \pm 1.2$ & $0.036 \pm 0.02$ & $1.88 \pm 0.17$ & $11.5 \pm 5.4$ \\
\hline p-value & 0.3 & 0.98 & 0.5 & 0.1 \\
\hline $\begin{array}{l}\text { Lymph node } \\
\text {-ve }\end{array}$ & $3.2 \pm 1.1$ & $0.035 \pm 0.027$ & $1.86 \pm 0.17$ & $10.5 \pm 3.7$ \\
\hline$+\mathrm{ve}$ & $3.4 \pm 1.2$ & $0.039 \pm 0.022$ & $1.95 \pm 0.17$ & $15.3 \pm 12.5$ \\
\hline $\mathrm{p}$-value & 0.5 & 0.3 & $0.004^{*}$ & $0.002 *$ \\
\hline $\begin{array}{c}\text { Distant metastasis } \\
\text { M0 }\end{array}$ & $3.03 \pm 1.03$ & $0.044 \pm 0.027$ & $1.85 \pm 0.17$ & $11.6 \pm 7.02$ \\
\hline M1 & $3.8 \pm 1.2$ & $0.023 \pm 0.012$ & $1.96 \pm 0.16$ & $13.5 \pm 10.4$ \\
\hline $\mathrm{p}$-value & $0.001 *$ & $<0.001^{*}$ & $<0.001 *$ & 0.2 \\
\hline
\end{tabular}

*Significant.

tumors compared with normal breast tissues [22]. Also, Heneghan and colleagues demonstrated that miR195 is significantly increased in the blood of breast cancer patients in comparison to disease-free control subjects, and is able to discriminate breast cancer patients from healthy controls with high specificity and sensitivity [3].

Furthermore, we analyzed the correlation between miR10b, miR34a, miR155 and miR195 expression and the clinicopathological data. MiR10b and miR155 were significantly up regulated in higher malignancy grades compared with lower grade ones. Wang and co-authors reported that higher tumor grades may show significantly higher expression of miR155 [20]. Using primary breast cancer tissues and epithelial cells, Kong and colleagues observed that miR155 can play an important role in the transforming growth factor- $\beta$ induced epithelial to mesenchymal transition (TGF- $\beta$-induced EMT), cell migration and cell invasion by targeting the small G-protein RhoA. They indicated that it might be a potential therapeutic target for breast cancer intervention [23].

miRNA34a was significantly down-regulated in advanced stages compared with early stages, while miR155 was significantly up-regulated in advanced stages compared with early stages of our patients. In contrast to our results, Roth and colleagues revealed high miR34a levels in the blood of patients with advanced tumor stages [14].

Furthermore, we observed a significant increase in serum miR10b and miR155 levels in metastatic breast 
cancer patients, compared with non-metastatic cases, while miR34a was significantly decreased.

Our results were in agreement with Roth and colleagues who found that the levels of miR $10 b$ significantly correlated with the occurrence of metastasis but not with Heneghan \& his coworkers who observed a significant increase in tumor miR195 levels in metastatic breast cancers, and reported that this pattern was reflected in the circulation $[14,24]$. On the contrary, Roth and colleagues detected high miR34a levels in the blood of metastatic breast cancer patients [14].

Supporting our results, Ma et al. reported that miR10b specifically played an important role in the metastatic process but not in primary tumor development. They found this miR to be highly expressed in metastatic breast cancer cells, and its overexpression initiated invasion and metastasis in a combination of mouse and human cell models by indirectly activating the prometastatic gene RhoC [11].

Our observation that circulating miR10b is higher in ER negative disease was keeping with the findings of previous studies $[11,20,24]$ which reported that miR10b was highly correlated to the ER or PR expression; great differences were detected in miR expression levels in samples that are hormone receptor negative, this was a predictive factor for prognosis in patients with breast cancer.

Women with PR-positive tumors had higher miR155 expression than those with tumors that were PR negative [21]. In our study, we did not observe different serum miR155 levels between hormone receptor-positive and -negative patients.

A potential relationship between circulating miRNA levels, menopausal status, type of disease (in situ vs. invasive), LN and HER2/neu status was also investigated, but no statistically significant difference was identified for any of these parameters.

Results presented here showed significantly altered circulating levels of certain miRNAs (miR10b, miR34, miR155, and miR195) in breast cancer patients compared with healthy controls and a significant correlation between these miRNAs' expression and some clinicopathological data. However, future larger studies and advanced technologies are needed to confirm our findings and to further explore the existing potential of circulating miRNAs as novel biomarkers for breast cancer in order to be utilized in the clinical field.

\section{MATERIALS AND METHODS}

\section{Blood Collection and serum preparation}

Blood samples were collected from 170 Egyptian female participants, including 120 consecutive breast, pathologicaly confirmed breast cancer patients admitted to Surgery Department of Zagazig University Hospitals (Zagazig, Egypt). Patients who had received chemotherapy or radiotherapy in the preoperative period were excluded. Fifty apparently healthy, age and sex-matched individuals, comprised the control group.

Three milliliters of venous blood were collected from the antecubital fossa and placed in a serum separator tube gel. The blood was centrifuged at $1600 \mathrm{rpm}$ for $5 \mathrm{~min}$ and serum was transferred into $1.7 \mathrm{ml}$ eppendorf tubes, then another centrifugation step was done at high speed $12,000 \mathrm{rpm}$ for $15 \mathrm{~min}$ to remove cell debris completely, leaving only circulating RNA.

A written informed consent was obtained from all of the study participants.

\section{Tissue samples preparation}

As regards tissue samples, four $\mu \mathrm{m}$ thick sections from formalin-fixed, paraffine-embedded tissue blocks were stained with hematoxylin-eosin for morphological assessment. Tumors were evaluated for tumor grading using the Elston and Ellis grading system for invasive carcinoma, and the criteria of the European Breast Screening Group for ductal carcinoma in situ (DCIS). Tumor staging was reported based on TNM, according to the World Health Organization (WHO 2003) classification of breast tumors $[25,26]$.

\section{Immunohistochemistry}

Immunohistochemical staining was carried out using streptoavidin-biotin immunoperoxidase technique (Dako-

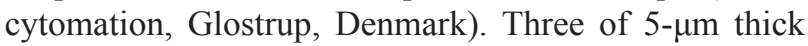
sections cut from formalin fixed paraffin embedded blocks were stained for each sample, and scoring was performed independently by two different expert observers. Hormone receptors were evaluated in the tissue samples using monoclonal antibodies: anti-ER (mouse monoclonal IgG, code number sc-56833, Santa Cruz Biotechnology, CA), anti-PR (rabbit polyclonal IgG, code number sc-539, Santa Cruz Biotechnology, CA), anti-HER2 (mouse monoclonal IgG, code number sc-33684, Santa Cruz Biotechnology, CA), and secondary antibodies. Product visualization was then performed employing diaminobenzidine (DAB) substrate chromogen (DakoCytomation,Glostrup, Denmark) .

\section{RNA Isolation}

RNA Isolation was done according to the manufacturer's instructions by using the miRNeasy Mini Kit (QIAGEN GmbH, Hilden, Germany) that combines phenol/guanidine-based lysis of samples and silica- 
membrane--based purification of total RNA. QIAzol Lysis Reagent, included in the kit, is a monophasic solution of phenol and guanidine thiocyanate, designed to facilitate lysis of tissues, to inhibit RNases, and also to remove most of the cellular DNA and proteins from the lysate by organic extraction.

\section{Conversion of RNA into cDNA (complementary DNA)}

RNA was reverse transcribed using miScript II RT Kit (QIAGEN GmbH, Hilden, Germany) according to the manufacturer's instructions. In each batch of reactions RT (reverse transcription) controls were included. Reverse transcription reactions were carried out in final volumes of $20 \mu \mathrm{l}$ using an AmpGene DNA thermal cycler 4800. Briefly, reactions consisted of $15-\mu 1$ RNA, 4- $\mu 1$ 5x miScript HiSpec Buffer containing nucleics mix and $1 \mu \mathrm{lmiScript}$ Reverse Transcriptase. Reactions were incubated for 60 min at $37^{\circ} \mathrm{C}$ then for $5 \mathrm{~min}$ at $95^{\circ} \mathrm{C}$ to inactivate miScript Reverse Transcriptase Mix. Finally, cDNA samples were Stored undiluted at $-80^{\circ} \mathrm{C}$.

\section{Analysis of miRNA Expression}

We chose to study a panel of four miRNAs in the circulation of our cancer patients (miR10b, miR34a, miR155 and miR195) based on their previously documented associations with malignancies [4, 27] and miR16 was used as an endogenous control to detect the effect of hemolysis [28]. Expression level of miRNAs was analyzed using spiked-in Syn-cel-miR-39 miScript miRNA Mimic (QIAGEN GmbH, Hilden, Germany) was chosen and added as the normalized internal control.

Real-time PCR quantification of mature miRNA or noncoding RNA was performed using target-specific miScript Primer Assays (forward primers) and the miScript SYBR Green PCR Kit, which contains the miScript Universal Primer (reverse primer), and QuantiTect SYBR Green PCR Master Mix (QIAGEN GmbH, Hilden, Germany) according to the manufacturer's protocol.

Before Real-time PCR, cDNA samples $(20 \mu \mathrm{l})$ were diluted by adding $100 \mu \mathrm{l}$ PCR grade distilled water (DW). PCR reactions were carried out in final volumes of $25 \mu \mathrm{l}$ using a StepOne ${ }^{\mathrm{TM}}$ System (Applied Biosystems). Briefly, reactions consisted of $2.5 \mu \mathrm{l}$ cDNA, $12.5 \mu \mathrm{l} 2 \mathrm{x}$ QuantiTect SYBR Green PCR Master Mix, $2.5 \mu$ 10x miScript Universal Primer, $2.5 \mu 1$ 10x miScript Primer Assay and $5 \mu \mathrm{l}$ PCR grade DW.

Reactions were initiated with a 15-minute incubation at $95^{\circ} \mathrm{C}$ to activate HotStarTaq DNA Polymerase followed by 40 cycles at $94^{\circ} \mathrm{C}$ for 15 seconds (denaturation), $55^{\circ} \mathrm{C}$ for 30 seconds (annealing) and $70^{\circ} \mathrm{C}$ for 30 seconds (extension\& fluorescence data collection). Each sample was duplicated and the mean of CT was calculated.
The relative expression of miRNA was calculated manually using the comparative cycle threshold $(\Delta \Delta \mathrm{Ct})$ method as follows: $\Delta \mathrm{Ct}=$ mean value $\mathrm{Ct}$ (miR of interest) - mean value $\mathrm{Ct}$ (reference $\mathrm{miR}$ ), $\Delta \Delta \mathrm{Ct}=\Delta \mathrm{CT}$ test sample $-\Delta \mathrm{CT}$ control sample.

The relative expression of $\mathrm{miR}$ of interest corresponded to the $2^{\wedge}(-\Delta \Delta C t)$ value [29], normalized to spiked-in Syn-cel-miR-39 levels. Then the relative expression levels of miRNA were confirmed by using free data analysis tools at http://pcrdataanalysis.sabiosciences. com/mirna.

\section{Statistical analysis}

Data was analyzed using SPSS statistical package version 17 (SPSS Inc., Chicago, IL). Independent “ $t$ ” test was used for quantitative values. A $p$-value $<0.05$ was considered significant.

\section{CONFLICTS OF INTEREST}

None declared

\section{GRANT SUPPORT}

This work was funded by an Academic Research Grant from Zagazig University Projects, Zagazig University Postgraduate \& Research Affairs.

\section{REFERENCES}

1. Parkin DM, Bray F, Ferlay J, Pisani P. Global cancer statistics. CA Cancer J Clin. 2002; 55: 74-108.

2. Allam MF, AbdElaziz KM. Evaluation of the level of knowledge of Egyptian women of breast cancer and its risk factors. A cross sectional study. J Prev Med Hyg. 2012; 53(4): 195-198.

3. Heneghan HM, Miller N, Kelly R, Newell J, Kerin MJ. Systemic miRNA-195 Differentiates Breast Cancer from Other Malignancies and Is a Potential Biomarker for Detecting Non invasive and Early Stage Disease. The Oncologist. 2010; 15:673-682.

4. Heneghan HM, Miller N, Lowery AJ, Sweeney KJ, Kerin MJ. MicroRNAs as Novel Biomarkers for Breast Cancer. J Oncol. 2010; Article ID 950201, 7 pages. http://dx.doi. org/10.1155/2010/950201

5. Iorio MV, Ferracin M, Liu CG, Veronese A, Spizzo R, Sabbioni S, Magri E, Pedriali M, Fabbri M, Campiglio M, Ménard S, Palazzo JP, Rosenberg A, Musiani P et al. MicroRNA gene expression deregulation in human breast cancer. Cancer Res. 2005; 65(16): 7065-70.

6. Li J, Smyth P, Flavin R, Cahill S, Denning K, Aherne S, Guenther SM, O'Leary JJ, Sheils O. Comparison of miRNA expression patterns using total RNA extracted from matched 
samples of formalin-fixed paraffin-embedded (FFPE) cells and snap frozen cells. BMC Biotechnol. 2007; 7:1-6.

7. Gilad S, Meiri E, Yogev Y, Benjamin S, Lebanony D, Yerushalmi N, Benjamin H, Kushnir M, Cholakh H, Melamed N, Bentwich Z, Hod M, Goren Y, Chajut A. Serum microRNAs are promising novel biomarkers. PLoS ONE. 2008; 3(9):e3148. doi: 10.1371/journal. pone. 0003148 .

8. VanSchooneveld E, Wouters MC, Van der Auwera I, Peeters DJ, Wildiers H, Van Dam PA, Vergote I, Vermeulen PB, Dirix LY, Van Laere SJ. Expression profiling of cancerous and normal breast tissues identifies microRNAs that are differentially expressed in serum from patients with (metastatic) breast cancer and healthy volunteers. Breast Cancer Res. 2012; 14(1): R34.

9. Wang J, Zhao H, Tang D, Wu J, Yao G, Zhang Q. Over expressions of MicroRNA-9 and MicroRNA-200c in Human Breast Cancers Are Associated with Lymph Node Metastasis. Cancer Biother Radiopharm . 2013; 28(4): 283 288.

10. Mattie MD, Benz CC, Bowers J, Sensinger K, Wong L, Scott GK, Fedele V, Ginzinger D, Getts R, Hagg C. Optimized high-throughput microRNA expression profiling provides novel biomarker assessment of clinical prostate and breast cancer biopsies. Mol Cancer. 2006; 5:24.

11. Ma L, Teruva-Feldstein J, Weinberg RA. Tumor invasion and metastasis initiated by microRNA-10b in breast cancer. Nature. 2007; 449:682- 668.

12. Mar-Aguilara F, Mendoza-Ram'ireza JA, Malag'onSantiagoa I, Espino-Silvad PK, Santuario-Facioc SK, RuizFloresd P, Rodr'1guez-Padillab C, Res'endez-P'ereza D. Serum circulating microRNA profiling for identification of potential breast cancer biomarkers. Disease Markers. 2013; 34:163-169.

13. Dutta KK, Zhong Y, Liu YT, Yamada T, Akatsuka S, $\mathrm{Hu}$ Q, Yoshihara M, Ohara H, Takehashi M, Shinohara T, Masutani H, Onuki J, Toyokuni S. Association of microRNA-34a overexpression with proliferation is cell type-dependent. Cancer Sci. 2007; 98:1845-1852.

14. Roth C, Rack B, Müller V, Janni W, Pantel K, Schwarzenbach H. Circulating microRNAs as blood-based markers for patients with primary and metastatic breast cancer. Breast Cancer Research. 2010; 12:R90.

15. Bommer GT, Gerin I, Feng Y, Kaczorowski AJ, Kuick R, Love RE, Zhai Y, Giordano TJ, Qin ZS, Moore BB, MacDougald OA, Cho KR, Fearon ER . p53- mediated activation of miRNA34 candidate tumor-suppressor genes. Curr Biol. 2007; 17:1298-1307.

16. Braun CJ, Zhang X, Savelyeva I, Wolff S, Moll UM, Schepeler T, Orntoft TF, Andersen CL, Dobbelstein M. p53-Responsive microRNAs 192 and 215 are capable of inducing cell cycle arrest. Cancer Res. 2008; 68:1009410104.
17. Javeri A, Ghaffarpour M, Taha MF, Houshmand $M$. Downregulation of miR-34a in breast tumors is not associated with either p53 mutations or promoter hypermethylation while it correlates with metastasis. Med Oncol. 2013; 30:413.

18. Volinia S, Calin GA, Liu CG, Ambs S, Cimmino A, Petrocca F, Visone R, Iorio M, Roldo C, Ferracin M, Prueitt $\mathrm{RL}$, Yanaihara N, lanza G, et al. A microRNA expression signature of human solid tumors defines cancer gene targets. Proc Natl Acad Sci USA. 2006; 103:2257-2261.

19. Liu J, Mao Q, Liu Y, Hao X, Zhang S, Zhang J. Analysis of miR-205 and miR-155 expression in the blood of breast cancer patients. Chin J Cancer Res. 2013; 25(1):46-54.

20. Wang F, Zheng Z, Guo J, Ding X. Correlation and quantitation of microRNA aberrant expression in tissues and sera from patients with breast tumor. Gynecologic Oncology. 2010; 119:586-593.

21. Zhu W, Qin W, Atasoy U, Sauter ER. Circulating microRNAs in breast cancer and healthy subjects. BMC Res Notes. 2009; 2:89.

22. Zhang H, Su B, Zhou QM, Lu YY. Differential expression profiles of microRNAs between breast cancer cells and mammary epithelial cells. Ai Zheng. 2009; 28:493- 499.

23. Kong W, Yang H, He L, Zhao JJ, Coppola D, Dalton WS, Cheng JQ. MicroRNA-155 is regulated by the transforming growth factor beta/ Smad pathway and contributes to epithelial cell plasticity by targeting RhoA. Mol Cell Biol. 2008; 28:6773-6784.

24. Heneghan HM, Miller N, Lowery AJ, Sweeney KJ, Newell J, Kerin MJ. Circulating microRNAs as novel minimally invasive biomarkers for breast cancer. Ann Surg. 2010; 251:499-505.

25. Elston CW, Ellis IO. Pathological prognostic factors in breast cancer. I. The value of histological grade in breast cancer: experience from a large study with long-term follow-up. Histopathology. 1991; 19:403-10.

26. Tavassoe 'li FA, Devilee P. Pathology and genetics: Tumours of the breast and female genital organs (WHO Classification of Tumors). IARC Press. 2003; p10.

27. Lowery AJ, Miller N, McNeill RE, Kerin MJ. MicroRNAs as prognostic indicators and therapeutic targets: Potential effect on breast cancer management. Clin Cancer Res. 2008; 14:360-365.

28. Kirschner MB, Kao SC, Edelman JJ, Armstrong NJ, Vallely MP, Van Zandwijk N, Reid G. Haemolysis during sample preparation alters microRNA content of plasma. PLoS One. 2011; 6 (9):e24145. Doi:10.1371/journal.pone.0024145.

29. Davoren PA, McNeill RE, Lowery AJ, Kerin MJ, Miller N. Identification of suitable endogenous control genes for microRNA gene expression analysis in human breast cancer. BMC Mol Biol. 2008; 9:76. 